\title{
Association of Nonalcoholic Fatty Liver Disease with Insulin Resistance in Type 2 Diabetes Mellitus - A Prospective Study
}

\author{
J alal MJ $\mathbf{A}^{1 *}$, Nisha $\mathbf{N}^{2}$, Basheer $\mathbf{S}^{2}$, J oseph $\mathbf{N}^{2}$ and \\ Shobha $\mathbf{P}^{2}$ \\ ${ }^{1}$ Department of Internal Medicine and Rheumatology, \\ VPS Lakeshore Hospital, Kochi, Kerala, India \\ ${ }^{2}$ Specialist, Department of Family Medicine, VPS \\ Lakeshore Hospital, Kochi, Kerala, India \\ *Corresponding author: Jalal MJ A, Department of \\ Internal Medicine and Rheumatology, VPS Lakeshore \\ Hospital, Maradu, Kochi, Kerala, India
}

Received: May 13, 2017; Accepted: J une 08, 2017; Published: June 15, 2017

\begin{abstract}
Background: Association of Nonalcoholic fatty liver disease (NAFLD) with type 2 diabetes mellitus (T2DM) is common. Our aim was to study the association of NAFLD with Insulin Resistance in T2DM patients.
\end{abstract}

Materials and Methods: Our study population included 100 patients of T2DM attending the medical out patient department of a Tertiary care center in Kochi. Presence of fatty liver in these patients was determined by abdominal sonography. Insulin Resistance was assessed by Homeostasis Model Assistant - Insulin Resistance (HOMA-IR). P-value less than 0.05 was considered statistically significant.

Results: The study group $(n=100)$ was divided into a NAFLD group $(n=45)$ and a non-NAFLD group $(n=55)$. The prevalence of NAFLD in our study was $45 \%$. The prevalence of obesity (measured by BMI), central obesity (measured by waist circumference and waist hip ratio) was higher in NAFLD group with increased values of $\mathrm{HbA1c}$ and triglyceride levels. Insulin Resistance was significantly higher in NAFLD group than Non NAFLD group ( $P$ value 0.02 , 0.02).

Conclusion: The prevalence of NAFLD is high in patients of Type 2 DM. Presence of NAFLD among T2DM patients is significantly associated with Insulin resistance.

Keywords: NAFLD; Insulin Resistance; Type 2 diabetes mellitus

\section{Introduction}

Nonalcoholic fatty liver disease (NAFLD) is a pandemic worldwide. NAFLD denotes a spectrum of changes occurring in the liver of non-alcoholic individuals. It is an emerging chronic liver disease [1]. Macrovesicular hepatic steatosis is the characteristic histological finding. NAFLD co-exists with features of the metabolic syndrome including obesity, type 2 diabetes mellitus [T2DM], dyslipidemia and hypertension. Hyperinsulinemia and increased free fatty acid delivery to the liver contributes to the pathophysiology of NAFLD [2]. NAFLD in T2DM increases the rates of cirrhosis and mortality and hence have a poor prognosis [3].

\section{Materials and Methods}

Our study was carried out in the Departments of Internal Medicine and Family Medicine, VPS Lakeshore Hospital, Kochi from January 2015 to October 2016. Initial screening was carried out to include/exclude the patients based on history taking and clinical examination. A total of 100 patients with age more than 18 years were included who were having diabetes of minimum 1-year duration. Patients who consumed alcohol, patients with other liver diseases such as malignancies, hepatitis, liver abscesses, and patients with deranged hepatic functions due to any other febrile illnesses/disease were excluded from the study.
A detailed history regarding the disease was taken, and complete physical examination was performed. Obesity was calculated with BMI [body mass index], whereas waist/hip ratio was measured as an index of splanchnic fat accumulation. After an overnight fast, serum samples were obtained from all subjects for liver function tests (aminotransferases and alkaline phosphates), fasting lipid profile (total cholesterol, triglycerides, lipoproteins), fasting blood glucose (FBS), HbA1C, and fasting insulin levels.

Homeostasis Model Assistant-Insulin Resistance (HOMAIR) was calculated as measure of insulin resistance using following formula:

HOMA-IR $=[$ fasting insulin $(\mu \mathrm{U} / \mathrm{ml}) \quad \times$ fasting glucose $(\mathrm{mmol} / \mathrm{l})] / 22.5$

Presence of fatty liver was determined by abdominal ultrasonography findings (diffuse increase in echogenicity as compared to that of the spleen or renal cortex). Patients were categorized as those with NALFD and those without NALFD. Statistical analysis was carried out for study parameters between the two groups (NAFLD and non-NAFLD) using student's $t$-test. $P<$ 0.05 was considered significant.

\section{Results}

The mean age of the patient was 55.02 years. Out of 100 patients,
J Fam Med - Volume 4 Issue 4 - 2017

ISSN : 2380-0658 | www.austinpublishing group.com

Jalal et al. (C) All rights are reserved
Citation: Jalal MJA, Nisha N, Basheer S, Joseph N and Shobha P. Association of Nonalcoholic Fatty Liver Disease with Insulin Resistance in Type 2 Diabetes Mellitus - A Prospective Study. J Fam Med. 2017; 4(4): 1119. 
Table 1: Demographic, anthropometric, and biochemical characteristics of 100 patients with T2DM.

\begin{tabular}{|c|c|c|c|}
\hline Parameter & Mean & Range & SD \\
\hline Age ( Years) & 55.02 & $19-88$ & 13.47 \\
\hline BMI (Kg/m²) & 28.12 & $17.2-36.05$ & 4.03 \\
\hline Waist hip Ratio & 0.95 & $0.78-1.01$ & 0.076 \\
\hline ALT (IU/L) & 19.9 & $1-72$ & 13.16 \\
\hline AST (IU/L) & 22.1 & $1-93$ & 13.1 \\
\hline Cholesterol (mg/dl) & 180.6 & $69-273$ & 38.1 \\
\hline HDL (mg/dl) & 42.2 & $22-65$ & 6.8 \\
\hline LDL (mg/dl) & 125.7 & $24-190$ & 35.5 \\
\hline Triglyceride (mg/dl) & 173.1 & $53-390$ & 55.1 \\
\hline HOMA-IR & 4.4 & $0.7-43.5$ & 4.9 \\
\hline FBS (mg/dl) & 117.5 & $67-319$ & 33.6 \\
\hline Insulin ( $\mu \mathrm{U} / \mathrm{dl})$ & 17.5 & $3-70$ & 8.7 \\
\hline HbA1C (\%) & 8.3 & $5.8-12.6$ & 1.3 \\
\hline
\end{tabular}

$47(47 \%)$ were males and $53(53 \%)$ were females. None of the subjects had history of alcohol consumption. Of 100 patients with T2DM, 55 (55\%) were found to have changes of fatty liver disease in abdominal ultrasonography examination. 32 males (32\%) and 33 females (33\%) had fatty liver disease. Elevated ALT ( $>40 \mathrm{IU} / \mathrm{dl})$ was seen in 10 patients $(10 \%)$ while 5 patients $(5 \%)$ had AST $>40 \mathrm{IU} / \mathrm{dl}$ (Table 1 ). Mean BMI was $28.12 \mathrm{~kg} / \mathrm{m}^{2}$ (17.2-37.05) (Table 1). BMI, waist/hip ratio, S. triglyceride level were significantly high (Table 2 ) in NAFLD group as compared to Non-NAFLD group $(P=0.009,0.0002,0.003$, respectively). Quantitative measures of insulin resistance - $S$. fasting insulin, HOMA-IR score showed significant association of NAFLD with increased insulin resistance.

\section{Discussion}

In our study, $55 \%$ of Type 2 diabetes mellitus had NAFLD based on abdominal ultrasound examination. This correlates with other studies that have reported the prevalence of NAFLD among DM patients at approximately 50\% (range: $21-78 \%$ ) [4]. No significant differences in sex distribution were noted. This is in contrast to previous studies where the prevalence of NAFLD among women was found to be higher than men. In recent studies, it has been suggested that both sexes might be afflicted equally [5].

BMI was significantly higher in patients with NAFLD (28.17 \pm 3.77) than those without NAFLD $(25.19 \pm 4.02)[P=0.007]$. Association of Obesity with NAFLD has been reported in various other studies [5]. Waist/hip ratio was significantly different between the two groups $(P=0.003)$. It denotes abdominal fat distribution. In 1993, Kral et al. observed significant correlation between waist/ hip ratio and the degree of hepatic steatosis, in patients with normal BMI [6]. Fatty liver and visceral fat are responsible for adiposityrelated to the pathogenesis of Insulin Resistance [7]. Organ-specific deposition of fat is a strong predictor of hyperinsulinemia and/or insulin resistance. Increased intra-myocellular triglyceride content correlates with muscle insulin resistance analogous to fat in the liver. Intra-myocellular triglyceride content is assessed via muscle biopsy or magnetic resonance imaging/computed tomography.
Table 2: Analogy of parameters between NAFLD and Non-NAFLD diabetic patients.

\begin{tabular}{|c|c|c|c|}
\hline Parameter & NAFLD & Non NAFLD & $\boldsymbol{p}$-value \\
\hline Age (Yr.) & $55.93( \pm 12.97)$ & $52.54(13.8)$ & $0.3(\mathrm{NS})$ \\
\hline BMI (Kg/m²) & $28.17(3.77)$ & $25.19(4.02)$ & 0.007 \\
\hline Waist Hip Ratio & $0.83(0.06)$ & $0.77(0.07)$ & 0.003 \\
\hline AST (IU/L) & $19.64(15.60)$ & $14.69(9.64)$ & $0.07(\mathrm{NS})$ \\
\hline ALT (IU/L) & $18.8(15.86)$ & $13.97(10.08)$ & $0.09(\mathrm{NS})$ \\
\hline Cholesterol (mg/dl) & $192.18(36.9)$ & $185.7(39.04)$ & $0.3(\mathrm{NS})$ \\
\hline HDL (mg/dl) & $41.03(7.02)$ & $16.42(6.66)$ & $0.8(\mathrm{NS})$ \\
\hline LDL (mg/dl) & $125.32(37.3)$ & $118.43(33.64)$ & $0.4(\mathrm{NS})$ \\
\hline Triglyceride (mg/dl) & $201.7(63.4)$ & $169.9(42.62)$ & 0.002 \\
\hline HOMA-IR & $5.36(6.79)$ & $3.2(1.99)$ & 0.029 \\
\hline FBS (mg/dl) & $121.1(41.8)$ & $111(24.02)$ & $0.08(\mathrm{NS})$ \\
\hline Insulin ( $\mu \mathrm{U} / \mathrm{dl})$ & $16.72(11.13)$ & $12.57(5.65)$ & 0.03 \\
\hline HBA1C (\%) & $8.27(1.39)$ & $7.93(1.18)$ & $0.31(\mathrm{NS})$ \\
\hline
\end{tabular}

Transaminase levels did not show statistically significant relationship between the NAFLD and non-NAFLD groups $(P=0.07$ and 0.09 for AST and ALT, respectively). In 2005, Adams LA et al suggested normal transaminases in up to $78 \%$ of NAFLD patients at any time, even when complete histological findings are present [8], explaining a poor correlation between transaminase levels and disease severity.

Mean cholesterol, HDL, and LDL levels did show significant difference between the two groups. Mean triglyceride levels showed statistically significant correlation with the presence of NAFLD $(P=0.02)$. As stated by previous studies on NAFLD, $20-92 \%$ of patients diagnosed with NAFLD have hyperlipidemia [4], including hypertriglyceridemia, hypercholesterolemia or both [5]. In 2000, Assy $\mathrm{N}$ et al. described hyperlipidemia in almost $50 \%$ of the patients who had NAFLD on ultrasound evaluations, of which only hypertriglyceridemia and not hypercholesterolemia was shown to pose a risk of developing liver fatty disease [9]. In 2002, Saadeh S et al. concluded that hypertriglyceridemia along with diabetes and obesity increases the risk of NAFLD development [10].

Glycemic control in terms of fasting glucose as well as HbA1C in NAFLD patients were not statistically significant as compared to nonNAFLD ( $P=0.08$ and 0.31 respectively). This observation suggests a non-causal relationship between glycemic control and fatty liver. When the triglyceride storing capacity of the fat cell is exceeded, fat overflows to other tissues like muscle and liver. This intracellular triglyceride metabolism interferes with insulin signaling, glucose transport and glycogen synthesis in muscle and increases hepatic gluconeogenesis.

Our patients in the NAFLD group had a higher prevalence of Insulin resistance as measured by S. fasting insulin and HOMA-IR; as compared to non-NAFLD group ( $P-0.03$ and 0.029 , respectively). Hyperinsulinemia itself may trigger hepatic fat deposition. This is empirically demonstrated by the hepatic steatosis occurring under the capsule of livers in patients undergoing peritoneal dialysis [11] where insulin is routinely added to the dialysate. 
NAFLD can be treated targeting obesity, insulin resistance, hyperlipidemia, and hypertension [12]. NAFLD in our study was not confirmed by liver biopsy, and this is a major limitation of our study. Only liver biopsy can assess the severity of damage and the prognosis of NAFLD. Ultrasonography is by far the most common method of diagnosing NAFLD in clinical practice. In 1991, Joseph AE et al concluded that ultrasonography has a very good sensitivity and specificity in detecting moderate and severe steatosis in patients with the biopsy-proven disease [13].

\section{Conclusion}

Early screening of NAFLD is advocated in regard to high incidence of NAFLD in T2DM patients. Moreover, our study correlates insulin resistance with the presence of NAFLD in diabetics. Large cohort studies are necessary to validate our results.

\section{References}

1. Browning JD, Szczepaniak LS, Dobbins R, Nuremberg P, Horton JD, Cohen JC, et al. Prevalence of hepatic steatosis in an urban population in the United States: impact of ethnicity. Hepatology. 2004; 40: 1387-1395.

2. Marchesini G, Brizi M, Morselli-Labate AM, Bianchi G, Bugianesi E, McCullough AJ, et al. Association of nonalcoholic fatty liver disease with insulin resistance. Am J Med. 1999; 107: 450-455.

3. Stranges S, Dorn JM, Muti P, Freudenheim JL, Farinaro E, Russell M, et al Body fat distribution, relative weight, and liver enzyme levels: A populationbased study. Hepatology. 2004; 39: 754-763.
4. Angulo P. Nonalcoholic fatty liver disease. N Engl J Med. 2002; 346: 1221 1231.

5. Reid AE. Nonalcoholic steatohepatitis. Gastroenterology. 2001; 121: 710 723.

6. Kral JG, Schaffner F, Pierson RN Jr, Wang J. Body fat topography as an independent predictor of fatty liver. Metabolism. 1993; 42: 548-551.

7. Gastaldelli A, Miyazaki Y, Pettiti M, Matsuda M, Mahankali S, Santini E, et al. Metabolic effects of visceral fat accumulation in type 2 diabetes. J Clin Endocrinol Metab. 2002; 87: 5098-5103.

8. Adams LA, Angulo P. Recent concepts in non-alcoholic fatty liver disease. Diabet Med. 2005; 22: 1129-1133.

9. Assy N, Kaita K, Mymin D, Levy C, Rosser B, Minuk G. Fatty infiltration of liver in hyperlipidemic patients. Dig Dis Sci. 2000; 45: 1929-1934.

10. Bayard M, Holt J, Boroughs E. Nonalcoholic fatty liver disease. Am Fam Physician. 2006; 73: 1961-1968

11. Wanless IR, Bargman JM, Oreopoulos DG, Vas SI. Subcapsular steatonecrosis in response to peritoneal insulin delivery: A clue to the pathogenesis of steatonecrosis in obesity. Mod Pathol. 1989; 2: 69-74.

12. McCullough AJ. The clinical features, diagnosis and natural history of nonalcoholic fatty liver disease. Clin Liver Dis. 2004; 8: 521-533.

13. Joseph AE, Saverymuttu SH, al-Sam S, Cook MG, Maxwell JD. Comparison of liver histology with ultrasonography in assessing diffuse parenchymal liver disease. Clin Radiol. 1991; 43: 26-31.
J Fam Med - Volume 4 Issue 4 - 2017

ISSN : 2380-0658 | www.austinpublishinggroup.com

Jalal et al. (C) All rights are reserved
Citation: Jalal MJA, Nisha N, Basheer S, Joseph N and Shobha P. Association of Nonalcoholic Fatty Liver Disease with Insulin Resistance in Type 2 Diabetes Mellitus - A Prospective Study. J Fam Med. 2017; 4(4): 1119. 